\title{
Interculturalización de la Educación superior en Nicaragua: del pensamiento teórico a la realidad
}

\author{
Interculturalization of Higher Education in Nicaragua: from theoretical thinking to reality
}

\author{
Ilenia Arllery García Peralta'
}

\section{Resumen}

En este artículo se evidencian importantes logros en la aprobación de normas y políticas que reconocen el derecho de los pueblos en términos de educación intercultural, y en específico en educación superior intercultural; sin embargo, las brechas entre lo aprobado y lo implementado todavía son amplias. Especial atención requiere la construcción de planes y programas académicos pertinentes, que refleje el espíritu de los pueblos indígenas y afrodescendientes; porque, aunque hay evidentes esfuerzos en la implementación de acciones afirmativas, el acceso y permanencia de jóvenes en las aulas de clase no es suficiente, todavía se requiere de cambios profundos para una verdadera interculturalización de la educación superior; porque el reto no es incluir a indígenas y afrodescendientes en las Instituciones de educación superior, sino transformarlas para que sean más pertinentes, inclusivas y equitativas.

Palabras clave: Acceso; educación superior; interculturalización; pueblos originarios; pueblos afrodescendientes.

\begin{abstract}
This article shows important achievements in the approval of norms and policies that recognize the right of peoples in terms of intercultural education, and specifically in intercultural higher education; however, the gaps between the approved and the implemented policies are still wide. Special attention requires the construction of relevant academic plans and programs that reflect the spirit of indigenous and Afrodescendant peoples; even though there are obvious efforts in the implementation of affirmative actions, the access and permanence of young people in classrooms is not enough, profound changes are still required for a true interculturalization of higher education; because the challenge is not to include indigenous and Afro-descendants in Higher Education Institutions, but to transform them so they can be more relevant, inclusive and equitable.
\end{abstract}

Keywords: Access; Higher Education; Interculturalization; originative peoples; afrodescendent peoples.

\section{Introducción}

La experiencia en educación superior para pueblos indígenas y afrodescendientes va en continuo crecimiento a nivel mundial, cuyo interés se deriva del deseo de "preservación cultural, que está vinculado a la autodeterminación, autonomía, territorio y soberanía" (Mato 2018 p: 48). El tema sobre el acceso a la educación intercultural y específicamente a la educación superior para pueblos indígenas y afrodescendientes ha venido tomando auge desde hace unas dos décadas. Varios son los actores que han estado involucrados

\footnotetext{
1 Máster en Gerencia Estratégica. Coordinadora de Cooperación Externa de la Universidad de las Regiones Autónomas de la Costa Caribe Nicaragüense- Recinto Universitario Bluefields. Email: ilenia.garcia@uraccan.edu.ni, ORCID: https://orcid.org/0000-0002-6152-8779
} 
en estos procesos, desde líderes y lideresas pertenecientes a estos pueblos, hasta otras personalidades que de forma individual o por medio de organizaciones gubernamentales y no gubernamentales trabajando en este importante campo. Los avances se evidencian en diferentes artículos que han sido publicados en congresos, foro - panel, revistas, entre otros, siendo uno de los espacios de mayor relevancia, la Conferencia Regional de Educación Superior, desde su inicio en el año 2006 hasta la actualidad.

En este documento se presenta un análisis de la información extraída de diferentes artículos que fueron sistematizados con la finalidad de dar a conocer/visibilizar/describir la experiencia de la educación intercultural en lo general y la educación superior en lo particular, en el contexto de los pueblos indígenas y afro descendientes, identificando las luchas y los avances que se alcanzaron a lo largo de los últimos veinte años. Partiendo de la información revisada se da salida a interrogantes como: ¿Cuáles han sido las oportunidades de acceso a la educación intercultural pertinente al contexto de pueblos indígenas y afrodescendientes? ¿Cómo se han materializado estas oportunidades? ¿Cuáles son los desafíos, tensiones y tendencias de la educación intercultural para estos pueblos?, partiendo de las tendencias, ¿se podrá interculturalizar la educación en Nicaragua? Dicho análisis es complementado con la experiencia de la Universidad de las Regiones Autónomas de la Costa Caribe Nicaragüense, una universidad comunitaria Intercultural, que fue soñada y creada por líderes y lideresas indígenas, afrodescendientes y mestizos caribeños de Nicaragua.

En general se evidencian importantes logros en la aprobación de normas y políticas que reconocen el derecho de los pueblos en términos de educación intercultural, y en específico en educación superior intercultural; sin embargo, las brechas entre lo aprobado y lo implementado todavía son amplias. Otros importantes avances están relacionados al desarrollo de programas de inclusión de individuos por medio de becas, asesoría académica y psicológica, programas especiales orientados a la formación de educadores, en cuyos planes buscan incluir los idiomas, conocimientos y métodos de aprendizaje y producción de conocimientos. También se evidencian avances en la creación de proyectos de docencia, investigación y vinculación social; de igual manera se han promovido el establecimiento de alianzas entre universidades y organizaciones y la creación de dos universidades interculturales, comunitarias, indígenas; siendo estas la Universidad de las Regiones Autónomas de la Costa Caribe Nicaragüense (URACCAN) y la Bluefields Indian \& Caribbean University (BICU).

En el proceso de la interculturalización de la educación, especial atención requiere la construcción de planes y programas académicos pertinentes, que reflejen el espíritu de los pueblos indígenas y afrodescendientes; aunque hay evidentes esfuerzos en la implementación de acciones afirmativas, el acceso y permanencia de jóvenes en las aulas de clase no es suficiente, todavía se requiere de cambios profundos para una verdadera interculturalización de la educación superior; porque el reto no es incluir a indígenas y afrodescendientes en las IES, sino transformarlas para que sean más pertinentes e inclusivas.

\section{Materiales y métodos}

El presente artículo de revisión bibliográfica es de carácter descriptivo - exploratorio. Para su construcción se realizó una revisión exhaustiva de unos veinticinco artículos, en los cuales diferentes autores hacen referencia sobre el trabajo que se ha venido desarrollando a lo largo de los últimos veinte años para mejorar el acceso a la educación intercultural, pertinente al contexto de pueblos indígenas y afrodescendientes.

\section{a. Tipos de materiales y método de búsqueda}

Se realizó revisión sistemática de artículos escritos por diferentes autores, entre estos Daniel Mato, Alvaro Guaymás, María Eugenia Choque, Rita Gómes, Xinia Zúñiga, Maribel Mora, Milena Mazabel, Luis Alberto Tuaza, Sergio Hernández, Inés Olivera, esteban Emilio Mozonyi, Alta Hooker, Libio Palechor, Anny 
Ocoro, Maria Da Silva, Luis Fernando Sarango, Jaime Zambrana y Marcia Mandepora. De igual manera se revisaron documentos que forman parte del compendio de normas y políticas de la universidad de las Regiones Autónomas de la Costa Caribe Nicaragüense (URACCAN), entre esos, el proyecto educativo institucional, el Modelo Pedagógico. Otros instrumentos jurídicos y técnicos fueron tomados en cuenta, como lo es La Constitución Política de Nicaragua, la Ley de Educación (Ley 582), así como las declaraciones de la Conferencia Regional de Educación Superior (1996, 2006, 2018), entre otros documentos de interés, vinculados a la temática. Todos ellos han compartido experiencias sobre la educación en lo general y la educación superior en particular y el acceso de pueblos indígenas y afrodescendientes, haciendo un análisis desde diferentes contextos.

En su mayoría los artículos han sido proporcionados como bibliografía base para desarrollar el segundo módulo del Doctorado en estudios Interculturales, ofertado por la Universidad de las Regiones Autónomas de la Costa Caribe Nicaragüense,

\section{b. Extracción y análisis de los datos}

Se realizó lectura completa de cada uno de los escritos, extrayendo lo vinculado a las líneas de abordaje de este artículo, las cuales responden a las interrogantes que nos hemos planteado. El análisis fue estructurado en cuatro grandes líneas a saber: i. El Contexto histórico que permitió visibilizar la lucha y pervivencia de los pueblos indígenas y afrodescendientes en la Costa Caribe de Nicaragua, por una educación inclusiva ii. La Educación Superior, pueblos indígenas y afrodescendientes: Experiencias, aciertos y desaciertos, iii. Los Desafíos, tensiones y tendencias en curso en el campo de Educación Superior; finalizando con un aporte que integra la percepción de diferentes autores sobre el camino hacia una verdadera interculturalización del sistema educativo en Nicaragua.

\section{Resultados y discusión}

\section{a. Contexto histórico: Lucha y pervivencia por el acceso a la educación intercultural, de los pueblos indígenas y afrodescendientes en la Costa Caribe de Nicaragua.}

Desde los tiempos de la conquista donde nuestros pueblos fueron masacrados y despojados de sus territorios, la lucha ha sido enérgica y constante; donde las costumbres, lenguas y religión fueron prohibidas, violentando de esta manera elementos constitutivos de su visión como pueblos (Mato, 2018, p. 17). A través de los años, la condición de estos pueblos fue cambiando, ya no eran esclavos, sin embargo las políticas de educación y cultura que implementaban los gobiernos de turno, en su afán de homogenización, les obligó a mantenerse marginados e invisibilizados; muchos perdieron su cultura, su lengua y hasta sus creencias. Fue una forma de esclavitud solapada.

"La educación es la base para el desarrollo de un pueblo" es una frase que se ha utilizado por muchos años en el afán de capturar el interés de las personas para que ingresen a las escuelas, colegios y universidades; sin embargo y pese a ser un derecho fundamental mandatado en distintos instrumentos internacionales, así como en la constitución política y leyes conexas, de los países latinoamericanos y específicamente en la de Nicaragua (arto. 58, 116,117,121 Cn), no siempre el acceso a la educación ha sido fácil, hay limitantes de infraestructura, cobertura, idioma, calidad, entre otros.

En diferentes documentos encontramos plasmada la historia de lucha por las reivindicaciones de los pueblos indígenas y afrodescendientes de la Costa Caribe nicaragüense, por su derecho a una educación de calidad, pertinente y relevante a su realidad multilingüe, multiétnica y pluricultural. A través de estas luchas ideadas y lideradas por costeños y costeñas, en los "últimos 30 años han logrado que el estado reconozca 
sus culturas, lenguas, modelo de salud, educación, cosmovisiones, formas de organización, identidad, tierra y territorio, mediante el Régimen de Autonomía Regional, aprobado por la Asamblea Nacional en 1987" (Hooker-Blandford, 2018, p. 308). Es así, apegados al derecho legalmente reconocido, que nace la Universidad de las Regiones Autónomas de la Costa Caribe Nicaragüense (URACCAN) y la Bluefields Indian and Caribbean University (BICU), ambas comunitarias e interculturales.

Las dos universidades desde su creación han facilitado el acceso a la educación superior y la formación profesional de hombres y mujeres costeñas/os; aportando de esta manera al fortalecimiento de la autonomía regional, mejoramiento de las condiciones de vida de los pueblos y comunidades, combatiendo el racismo, la exclusión y discriminación. De igual manera se ha facilitado la apertura de espacios de participación y toma de decisión para líderes y lideresas de los pueblos indígenas, afrodescendientes y comunidades mestizas.

Entre otros aportes, es meritorio mencionar la creación misma del Subsistema Educativo Autonómico Regional (SEAR), el cual fue definido desde sus inicios en 1996 y en sus primeras propuestas de leyes y políticas como "un modelo educativo participativo, el cual se gestiona de manera descentralizada y autónoma y que responde a las realidades, necesidades, anhelos y prioridades educativas de su población multiétnica, multilingüe y pluricultural" (Arto.41 Ley General de Educación). Su fuerza jurídica está basada en la constitución de la República (1987 y reformas 2014), en la cual se reflejan los principios esenciales, así como en la Ley General de Educación, como uno de los cinco subsistemas del Sistema Educativo Nacional (Ley 582, aprobada en 2006), - siendo este marco legal uno de los más avanzados en América Latina y el Caribe- cabe señalar que este sub-sistema está orientado hacia la formación integral de niños y niñas, jóvenes y adultos, hombres y mujeres de la costa caribe, en todos los niveles del sistema educativo, así como hacia el respeto, rescate y fortalecimiento de sus diversas identidades étnicas, culturales y lingüísticas (EDUQUEMOS, 2015. p. 7).

El SEAR, está estrechamente vinculado a los demás subsistemas educativos, contemplados en la ley de educación, particularmente al subsistema de educación superior, el cual funciona como el último peldaño educativo a nivel del sector educación en Nicaragua. La vinculación es importante porque en las universidades públicas y privadas, son los espacios donde se profesionalizan los docentes que están en las aulas de clase; cuya formación debe ser pertinente y contextualizada a los requerimientos de la educación preescolar, primaria, secundaria y técnica. Pero, además son estos profesionales, quienes están encargados de construir, proponer y dar salida a los planes y programas propuestos por el SEAR, ante las autoridades educativas regionales y nacionales.

En el 2015, en el marco del Foro Educativo Nicaragüense EDUQUEMOS, se elaboró el informe de progreso educativo, cuya construcción contó una amplia participación de actores nacionales, regionales y locales. En este documento se evidencian importantes avances en la educación de la Costa Caribe, alcanzados desde la creación del SEAR, entre estos se mencionan: La instalación de las estructuras regionales (Secretaria regional de educación) y descentralización de algunas funciones; Procesos de planificación participativos, de los cuales surgieron el plan decenal y plan septuagenal (aun en vigencia); fortalecimiento de autoridades educativas regionales, municipales y comunales; readecuación curricular en diferentes niveles formativos, así como elaboración de textos y materiales didácticos para la educación intercultural bilingüe (EIB de primero a sexto grado) y una importante oferta académica de educación superior regional, vinculada a la EIB. Cabe señalar que a pesar de estos importantes avances, en el mismo informe se indica que en la actualidad hay un estancamiento del SEAR, siendo una de las principales limitantes la poca disponibilidad de recursos humanos, materiales y financieros en la Secretaria Regional de Educación, para impulsar las acciones contempladas en el plan septuagenal; acciones que dan salida al fortalecimiento del sistema y su regionalización; esto vinculado al desconocimiento del SEAR por parte de autoridades y funcionarios del 
Ministerio de Educación del nivel central, lo que genera una doble conducción institucional y poca voluntad política - técnica para acompañar el fortalecimiento del SEAR.

\section{b. Educación Superior en Nicaragua, pueblos indígenas y afrodescendientes: Experiencias}

\section{i. Programas de inclusión y de formación especial para individuos indígenas y afrodescendientes en universidades y otras IES2 convencionales}

En los diferentes artículos se han identificado experiencias de educación superior para pueblos indígenas y afrodescendientes que de alguna manera han contribuido a mejorar las posibilidades de acceso a la formación profesional. Estas experiencias van desde la creación de programas especiales de formación superior (licenciaturas, maestrías, educación continua); programas de becas dirigido a miembros de pueblos indígenas y afrodescendientes; alianzas estratégicas entre instituciones de educación superior mayormente convencionales y organizaciones indígenas, afrodescendientes y comunidades mestizas; y la creación de universidades/centros formativos indígenas, comunitarias e interculturales. De acuerdo a lo planteado por Mato (2018), en sus diferentes artículos - pues ha sido uno de los actores interculturales que ha trabajado la temática- puedo concretar que en Nicaragua al igual que otros países de Centroamerica y América Latina se han desarrollado diversas experiencias que responden a las tendencias generales y a los nuevos planteamientos hechos a las universidades convencionales, desde la cosmovisión de los pueblos indígenas y afrodescendientes de la Costa Caribe nicaragüense. Partiendo de esta experiencia, se evidencian dos grandes líneas de trabajo: Las acciones afirmativas desarrolladas por las universidades convencionales y otras IES y la creación de universidades comunitarias interculturales.

En Nicaragua existen formalmente 60 universidades, cinco públicas, dos comunitarias, indígenas, interculturales y el resto son privadas (Muñoz, 2018 p. 131). Desde las universidades públicas y privadas "Convencionales", se han venido desarrollando algunas acciones afirmativas. Desde los años 70, por gestión de un grupo de costeños y costeñas, se logró una extensión de la universidad Nacional Autónoma de Nicaragua, ubicándose en las cabeceras regionales Bluefields y Bilwi, ofertando cursos en Ciencias de la Educación. Estas iniciativas de programas de formación ${ }^{3}$ continuaron en los años 8o, lamentablementepor falta de financiamiento- los y las estudiantes no lograron culminar con sus estudios, pese al esfuerzo y dedicación (URACCAN, 2018, p. 9). Otras experiencias estaban relacionadas a la facilitación de cupos para estudiantes provenientes de pueblos indígenas y afrodescendientes de la Costa Caribe, para que ingresaran a las universidades ubicadas en el Pacífico de Nicaragua. De 170 costeños y costeñas que anualmente recibían becas, solamente entre el $3 \%$ y el $5 \%$, lograban graduarse. Algunas de las razones obedecían a la falta de asistencia económica, ya que la beca no cubría todas las necesidades que surgen cuando un estudiante debe vivir fuera de su región, aunque para la mayoría el problema fundamental era que no lograban adaptarse al entorno ofrecido en las universidades, las que carecían de un enfoque intercultural en las Instituciones de Educación Superior, terminando por abandonar sus estudios o salían en busca de trabajo, de marineros, y meseros en barcos extranjeros (URACCAN, 2016, p. 14).

Otra iniciativa que realizaban algunas universidades fueron los programas de asistencia especial y tutorías en asignaturas básicas como matemática y español, pues los estudiantes caribeños llevaban desventaja con respecto a otros procedentes del resto del país. Otra experiencia desarrollada por la Universidad Autónoma de Nicaragua, con sede en Managua, con fondos de cooperación externa, fue la ejecución de los programas de Educación Continua, dirigidos al fortalecimiento de docentes mayangnas, en el marco del programa de Educación Intercultural Bilingüe (PEBI); de igual manera se llevaron a cabo procesos de investigación

\footnotetext{
2 Instituciones de Educación Superior.

3 Programa Universitario de Educación a Distancia-PRUEDIS y en 1992 con el Centro Universitario de la Región Autónoma del Atlántico Norte-CURAAN.
} 
sobre el buen vivir, territorio, gobernabilidad y demarcación de las tierras de los pueblos indígenas, afro descendientes y comunidades étnicas del Pacífico y Caribe del país (Muñoz, 2018, p. 131).

Algunos actores manifiestan que las acciones afirmativas para facilitar acceso a la educación superior a los pueblos indígenas, afrodescendientes y comunidades étnicas, "ha sido vistas como procesos homogenizadores que favorecen la fuga de cerebros, pérdida de valores, leguas y saberes". Ciertamente estas acciones por insignificantes que parecieran, en su momento dieron la oportunidad a jóvenes indígenas, afro descendientes y mestizos costeños, de acceder a estudios de educación superior, por medio de los cuales lograron prepararse, y en el caso de algunos de ellos regresar a la región caribeña y dar inicio a la lucha por la reivindicación de el derecho a la educación superior. Otros, desafortunadamente se establecieron en diferentes departamentos del país o en el extranjero por motivos familiares o laborales.

\section{ii. La creación de Universidades Comunitarias Interculturales}

En los últimos 20 años, los pueblos indígenas y afrodescendientes han tenido un destacado protagonismo en el quehacer político y social en América Latina y el Caribe, donde se ha impulsado buena parte de los profundos cambios institucionales verificados en la región. Una acción de gran impacto ha sido la generación de Instituciones de Educación Superior Interculturales y Comunitarias, alternativas a las convencionales; con innumerables experiencias desarrolladas, ya sean como parte de un esfuerzo autónomo de las organizaciones indígenas, de líderes y lideresas o apoyadas por el Estado y sus gobiernos.

Los pueblos indígenas y afrodescendientes en Nicaragua han venido luchando por tener su propio modelo educativo, el cual responda a sus necesidades y expectativas. Uno de los mayores logros de la Costa Caribe nicaragüense, en el marco de la Autonomía Regional, promulgada en 1987, cuando se aprueba el estatuto de autonomía (Ley 28), fue la creación y funcionamiento de dos universidades comunitarias interculturales: Universidad de las Regiones Autónomas de la Costa Caribe Nicaragüense (URACCAN) y la Bluefields Indian \& Caribbean University (BICU). Es por ello que líderes indígenas y afrodescendientes, con el acompañamiento de movimientos indígenas y otros actores interculturales se dieron a la tarea de crear universidades indígenas, afrodescendientes, interculturales y comunitarias. Ambas universidades han logrado establecer relaciones y coordinaciones pertinentes en el seno del Consejo Nacional de Universidades (CNU), como ente regulador de la educación superior en Nicaragua; con la finalidad de incidir en la interculturalización de la educación superior. De igual manera han establecido relaciones de trabajo con el Consejo Nacional de Evaluación y Acreditación (CNEA), con quienes han coordinado para la construcción de criterios y modalidades de evaluación participativa y de aprendizaje mutuo, consistente con la idea de interculturalidad (Hooker-Blandford, 2018, p. 308).

Gracias al sueño de un grupo de líderes y lideresas indígenas y afrodescendientes de la Costa Caribe, en 1992 se crea la URACCAN. Esto como respuesta a la demanda de formación profesional contextualizada y que respondiera a los sueños y aspiraciones de los pueblos de la Costa Caribe. Fue autorizada por el CNU en el mismo año, mediante decreto 602, publicado en La Gaceta No. 104 y en 1993, la Asamblea Nacional le otorga personería jurídica. Se inaugura el 30 de Octubre de 1994 y apertura sus puertas a la población costeña en 1995. Siendo un proyecto institucional de universidad propia que responde a las demandas y aspiraciones históricas de los pueblos indígenas, afrodescendientes y comunidades mestizas, en el año 2003, fue declarada Patrimonio de los Pueblos Indígenas y comunidades étnicas, por los consejos regionales autónomos de la Región Autónoma de la Costa Caribe Sur y Norte del país (Hooker-Blandford, 2018; Núñez 2018).

La BICU, se fundó en 1991 en el municipio de Bluefields, y recibió su personería jurídica en 1993. Se define como una universidad autónoma, comunitaria y de servicio público. Es apartidaría, multiétnica, 
intercultural, laica y sin fines de lucro. De igual manera fue reconocida por el Consejo Regional como universidad comunitaria intercultural. Ambas universidades caribeñas (URACCAN y BICU), cuentan con sedes a nivel de toda la Costa Caribe. Tienen financiamiento del 6\% constitucional, del presupuesto general de la Republica y forman parte del Consejo Nacional de Universidades. Cuentan con una matrícula aproximada de diecisiete mil estudiantes, una oferta académica con más de 40 carreras a nivel de licenciaturas, ingenierías y técnico superior; en un amplio campo de conocimientos como ciencias humanísticas y jurídicas, ciencia tecnológica y medio ambiente, ciencias médicas, ciencias de la educación, ciencias administrativas e informática. Otros procesos formativos como maestrías, posgrados y educación continua. En el caso de la URACCAN, ha iniciado el primer doctorado a nivel de la Costa Caribe y el primero en la temática de Estudios Interculturales a nivel de Nicaragua.

Sin un sistema de educación de calidad diseñado por nosotros y manejado por nosotros, que incluye un fuerte sistema de valores que preserva lo mejor de las prácticas de nuestros ancestros tales como lo sagrado de toda forma de vida, la vitalidad de la comunidad y la armonía con nuestro ambiente, en combinación con una base sólida en las ciencias y las humanidades, sin un sistema de esta naturaleza y con el control debido, no tenemos ninguna posibilidad de construir un mejor modo de vida para nuestro pueblo que asegure el éxito del proceso autonómico.

Dr. Ray Hooker (2001).

Lo que distingue a las universidades comunitarias interculturales de la Costa Caribe, es el espíritu del pueblo que se institucionaliza y dinamiza toda su actividad con una visión y misión propia (URACCAN, 2016, p. 12).

\section{iii. Colaboración Intercultural en investigación, docencia y servicio a las comunidades: Reflexiones}

La colaboración intercultural, vista desde la participación activa y propositiva de diferentes actores, ha sido fundamental, en principio para facilitar el acceso a la educación superior de pueblos indígenas y afro descendientes, a través de distintas acciones afirmativas; $y$, posteriormente en la creación de universidades que responden a modelos de educación propio.

Es importante destacar el papel que han desempeñado algunos actores para lograr avances significativos, entre estos los movimientos indígenas y afrodescendientes, líderes indígenas y afro descendientes, universidades comunitarias interculturales, las agencias gubernamentales y no gubernamentales, las redes como espacios para la colaboración entre universidades, agencias de cooperación internacional, entre otros actores que de alguna manera han venido colaborando en esta lucha. Muchos continuarán desempeñando un rol preponderante y de seguro profundizarán en sus luchas, partiendo de lo ya alcanzado. Particularmente la URACCAN ha contado con una amplia colaboración por parte de organizaciones internacionales como: El fondo de asistencia internacional de los estudiantes y académicos noruegos (SAIH), Organización Austriaca de Cooperación para el Desarrollo (Horizont, 300o), Unión Europea, agencia Noruega de cooperación al desarrollo (Norad), Agencia de los Estados Unidos para el Desarrollo Internacional (USAID), OXFAM, Agencia Española de Cooperación Internacional para el Desarrollo (AECID); así como otras instituciones y organizaciones nacionales y regionales, entre los que menciono: Instituto Nacional Forestal, Ministerio del Ambiente y Recursos Naturales, Fundación para la Autonomía y el Desarrollo de la Costa Atlántica (FADCANIC), Centro de Derechos Humanos, Ciudadanos y Autonómicos (CEDEHCA), Consejo Regional, Gobierno Regional, Gobierno Territorial de Awaltara, Gobierno Territorial de la Cuenca Laguna de Perlas, 
Gobierno territorial del Pueblo Rama - Kriol, entre otros (URACCAN, 2018). Cabe señalar que los programas y proyectos de cooperación universitaria e internacionalización de la URACCAN, contribuyen a la transformación social desde las perspectivas del desarrollo con identidad, buen vivir, vivir con dignidad y la ciudadanía intercultural de género. Incluyen procesos de fortalecimiento de capacidades autonómicas del liderazgo comunitario y actores sociales regionales mediante diplomados comunitario, cursos de grado y postgrados (URACCAN, 2018, p. 36).

En la actualidad y partiendo de la experiencia antes mencionada, considero que los espacios de colaboración intercultural, han resultado ser importantes para desarrollar procesos de articulación de la docencia, investigación y la vinculación social comunitaria; los cuales surgen en un principio, del "diálogo de saberes", en igualdad de condiciones; porque no puede haber acción, producto o resultado sin que los actores/as se haya sentado a soñar, reflexionar, intercambiar y consensuar ideas para luego construir. En ese sentido, y con la finalidad de articular aprovechando las convergencias, la URACCAN se ha integrado en algunas redes como la Red de Universidades Indígenas, Interculturales y Comunitarias de Abya Yala (RUICCAY), el Consejo Superior de Universidades centroamericanas (CSUCA), La Unión de Universidades de América Latina y el Caribe (UDUAL), la Red sobre Internacionalización y Movilidades Académicas y Científicas (RIMAC), la Red INCA de Cooperación e Internacionalización de la Educación Superior en América Central, la Organización Negra Centro americana (ONECA), entre otras de importancia a nivel internacional (URACCAN, 2018, p. 41).

Las universidades necesitamos formar profesionales y técnicos que sean pertinentes con la diversidad cultural y con la existencia de diversas visiones del mundo, Mato (2018). Los docentes juegan un papel fundamental en la articulación de la teoría con la práctica; para ello debemos sacar a nuestros estudiantes de las aulas de clase; brindándoles la oportunidad de compartir con sabios y sabias desde su área de trabajo o comunidad. Esa es la manera de enseñarles que no existe la verdad absoluta y que el conocimiento científico con el saber tradicional se complementan. Es por ello que la URACCAN ha definido dentro del Modelo Pedagógico y del proyecto educativo institucional la necesidad de incorporar el diálogo de saberes y haceres y el reconocimiento de la diversidad de valores y modos de aprendizaje como elementos centrales de las políticas, planes y programas del sector educación.

Con respecto a la creación y recreación de conocimientos, saberes y prácticas, estamos claros que los pueblos indígenas y afrodescendientes, deben ser parte activa y no objetos; pues la integración de conocimientos y saberes enriquece los resultados de una investigación. Por otro lado, involucrar a los estudiantes en las tareas diarias de la comunidad, les da un panorama real de las formas de vida, que incluye la solución innovadora a problemas que se les presentan en diferentes ámbitos sociales.

Desde los programas y proyectos, la Universidad implementa también importantes procesos investigativos que aportan a la construcción, reproducción y enriquecimiento del conocimiento. Está orientado a la solución de los principales problemas de bienestar de la población multiétnica de las Regiones Autónomas. En el acompañamiento comunitario intercultural, se sustentan procesos de revitalización cultural, productiva, ambiental, mejora de la educación y salud, coadyuvando al fortalecimiento y profundización de la Autonomía Regional desde la perspectiva intercultural de género.

"No hay saber universal, la colaboración intercultural es imprescindible", es una frase escrita por Mato (2018), que me ha permitido reflexionar porque muchos actores creemos que en aquello que hacemos o decimos está la verdad absoluta. Mientras exista este pensamiento, también continuarán las luchas por aquellos que creemos en la existencia de una verdad relativa y que cada persona, grupo, pueblo o comunidad, tiene su propia concepción de la vida, la naturaleza y el mundo. No podemos homogenizar el conocimiento, debemos aprender a reconocer y valorar las diferencias culturales y desarrollar un diálogo honesto, 
respetuoso, de mutuo interés, que parta de reconocer que hay diversidad de contextos, pensamientos y prácticas. Promover sociedades de conocimientos, permitirá avanzar en la búsqueda del desarrollo con identidad, mediante la innovación y el emprendimiento.

\section{c. Desafíos, tensiones y tendencias en el curso de Educación Superior y los pueblos indígenas y afrodescendientes en Nicaragua}

Se avizora que a partir de los elementos considerados en la reciente declaración CRES 20184; se construyan o mejoren las políticas públicas en el ámbito de la educación de forma general y en la interculturalización de la educación superior, en lo particular. Partiendo de lo antes mencionado, se espera que las experiencias de educación superior con pueblos indígenas y afrodescendientes tomen mayor auge en los años venideros.

La lectura además de permitirnos valorar las experiencias, también nos ayuda a reconocer los desafíos, entre estos hago mención de los más evidentes:

a. Necesitamos continuar trabajando en la disminución del racismo, ya que este no siempre se presenta de formas abiertas, sino solapadas, institucionalizadas y hasta inconsciente, en la sociedad en general y en las universidades en lo particular, incluyendo las comunitarias interculturales.

b. Hay que incidir en la construcción de políticas públicas con plena participación de pueblos indígenas y afrodescendientes, de tal manera que se garantice el derecho en todos los ámbitos sociales. Se debe aprovechar el amplio marco legal que respalda el derecho a los espacios de participación y toma de decisión en las estructuras regionales, municipales, territoriales y comunales.

c. Necesitamos sistemas de evaluación y acreditación que consideren la calidad, entendiendo como el bien hacer, asociada a la pertinencia cultural y relevancia. Cabe señalar que actualmente se cuenta con importantes avances, gracias al trabajo coordinado con el CNEA.

d. Reconocimiento, inclusión y asignación presupuestaria a universidades comunitarias interculturales. Aunque en Nicaragua las universidades comunitarias interculturales cuentan con este beneficio (6\% constitucional), se debe trabajar para aumentar el porcentaje asignado, pues actualmente de las diez universidades que conforman el Consejo Nacional de Universidades, BICU y URACCAN, reciben el menor porcentaje.

e. Visibilizar la sistematización y resultado de investigaciones (tesis, monografías, investigaciones libres), de las universidades comunitarias interculturales y otros actores que acompañan estas vivencias.

f. Mejorar las capacidades de construcción de relaciones de colaboración provechosa y duradera por medio de las redes.

g. Visibilización de pueblos indígenas y afrodescendientes en los censos poblacionales, ya que en la actualidad aunque se registran importantes avances todavía no reflejan la realidad de los pueblos en términos cuantitativos, lo que limita la inclusión en políticas públicas.

Estos desafíos concuerdan con los ejes de conflictos y tensiones que menciona Mato (2018), entre estos, la necesidad de sensibilizar a los actores que trabajan e implementan las políticas públicas; la creación de

${ }^{4}$ Conferencia Regional de Educación Superior. 
mecanismos y criterios de evaluación y acreditación pertinentes al contexto de las universidades indígenas interculturales; la transformación profunda de las universidades y otras IES convencionales; la asignación presupuestaria estatal, así como la articulación a lo interno y externo de los pueblos.

En base al contexto antes descrito, y luego de importantes sucesos como los alcanzados en la CRES (2018), se identifican algunas tendencias, que nos indican por donde debe continuarse el trabajo en la búsqueda de la interculturalización de la educación superior; entre estas; i. Prepararnos en el uso de las nuevas tecnologías de educación, ya que nos abre importantes posibilidades para avanzar en procesos formativos y de articulación; ii. La integración de redes a nivel centro americano y latinoamericano, para emprender luchas con mayor fuerza, la experiencia del trabajo en red evidencia la importancia de las mismas iii. Mayor posibilidad de integración en distintos espacios de participación, reflexión y toma de decisión a nivel internacional, oportunidades que deben ser aprovechadas para la incidencia y visibilización de los pueblos y sus demandas; v. Mayor posicionamiento de los pueblos indígenas y afrodescendientes a nivel de Centroamérica y Latinoamérica, vi. Surgimiento de otras universidades indígenas, comunitarias interculturales.

Pese a las oportunidades que se avizoran a futuro, también debe considerarse aquellas tendencias que de alguna manera limitarían el trabajo para la incidencia, posicionamiento y visibilización como lo es el cese de programas de colaboración económica por parte de las organizaciones internacionales de cooperación externa y organismos estatales. Ante esto, las universidades y organizaciones locales, debemos buscar alternativas para continuar con el trabajo de la interculturalización de la educación superior y el fortalecimiento de las estructuras de líderes/as indígenas, afrodescendientes y mestizos.

\section{d. Hacia una verdadera interculturalización de la educación superior de Nicaragua: Un panorama incierto}

A pesar de todos los importantes avances que se han mencionado en ítems anteriores, nos queda claro que todavía persisten retos en la construcción de una verdadera interculturalización de la educación como sistema y específicamente en la educación superior. Los diferentes artículos evidencian que la temática se manifiesta actualmente en todo el mundo, cargada de diversos contenidos y significaciones según quien la reivindique, la condene o la describa. Sin embargo, es importante resaltar que actualmente se ha convertido en una categoría de reflexión y análisis para algunas instituciones y plataformas académicas (Hooker 2018).

Para que haya una verdadera interculturalización de la educación, es necesario una transformación significativa de todo el sistema educativo, ya que aún persisten desigualdades, inequidades, conflictos y dificultades en la inclusión, desarrollándose imaginarios nacionales homogeneizantes (Hooker-Blandford, 2018). Debemos asumir con acciones concretas la interculturalización de la educación desde la inicial hasta la superior, como un eje central, distinto al modelo tradicional, haciendo transformaciones profundas en las estructuras, organizaciones, universidades e instituciones del país. Por eso debemos asumir que la interculturalidad es un asunto de Estado y no sólo un problema o un derecho de los indígenas y afrodescendientes, para ser ejercido en sus propias geografías o contextos sociales. No se trata de reproducir una educación que replique el sistema vigente, donde los conocimientos indígenas y afrodescendientes, en el mejor de los casos, sean un aspecto circunstancial en el currículo académico. Tampoco se pretende crear un espacio privilegiado del saber, reservado solamente para los indígenas y negros, sino de construir contenidos fundamentales que permitan vivenciar la interculturalidad, potenciando la riqueza de los saberes ancestrales, emprender un diálogo teórico práctico desde lo intercultural, lo que requiere la construcción de nuevos marcos conceptuales, analíticos, teóricos, en los cuales se vayan generando nuevos conceptos, categorías y nociones, desde la perspectiva de la interculturalidad, calidad y pertinencia.

La necesidad de interculturalización de la educación obliga a los gobiernos y sus funcionarios a reexaminar las políticas públicas estatales y su implementación en materia educativa, para que asuman la interculturalidad como columna vertebral del sistema, y no la homologación y jerarquización de conocimientos 
discriminadores y excluyentes (URACCAN, 2016). Por otro parte, debe haber un trabajo continuo con funcionarios que por su ubicación y cargos institucionales son claves para alcanzar la interculturalización en todo el sistema educativo del país, así como en el mismo sub sistema regional de educación; ya que muchos de estos no entiende su significado, lo que se persigue o simplemente su mismo racismo solapado, los convierte en barreras que detienen los procesos.

Considero que también es importante que se continúe promoviendo el diálogo intercultural, con los diversos actores del sistema de Educación (superior, media, inicial primaria y secundaria). Necesitamos que haya espacios para que se escuchen una amplia variedad y diversidad de voces, difundan las distintas formas de vida y compartan las distintas maneras de ver el mundo. Esto mejoraría los procesos de articulación de todo el sistema, y la responsabilidad en la construcción de ciudadanías interculturales ${ }^{5}$, no recaería únicamente sobre los docentes de educación superior. Es urgente el trabajo de sensibilización en valores interculturales, insertándolo desde la educación inicial.

\section{Conclusiones}

Es necesario un cambio de paradigma donde los pueblos indígenas y afrodescendientes sean puestos en el centro de las estrategias, como actores y partícipes de su desarrollo. Las universidades convencionales y las indígenas, interculturales y comunitarias, deben profundizar en el diálogo de saberes y haceres, en los modos de producción de conocimientos, modos de aprendizajes, lenguas, historias, espiritualidad, visiones del mundo; espacios donde la comunidad debe tener un amplio protagonismo. Para que haya mayor incidencia a nivel nacional, debe continuarse el trabajo de fortalecimiento del Sistema Educativo Autonómico Regional, en búsqueda de la regionalización del mismo. Para ello, la colaboración intercultural es fundamental, mediante la creación de espacios de dialogo, convergencias, cooperación; así como fortalecer la confianza mutua entre los diferentes actores de la educación intercultural.

La tarea inmediata que debemos emprender en todas las universidades es la interculturalización, entendida como un proceso de transformación institucional interna y externa que dé cuenta de la armonía que deben tener nuestras universidades con su entorno y su realidad económica, política, social y cultural. La experiencia ha demostrado que no es suficiente ser una universidad indígena, contar con presencia de estudiantes indígenas de diversas culturas, cuando las prácticas educativas y los contenidos temáticos occidentales convencionales siguen teniendo predominancia sobre los saberes propios de los pueblos indígenas y no se definen políticas concretas de fortalecimiento, de desarrollo, de investigación y de difusión de los saberes, conocimientos y lenguas, sino que se sigue reproduciendo la misma lógica de cualquier centro de formación (Sarango, 2009).

\section{Lista de referencia}

Carta de las Universidades e Instituciones de Educación de los Pueblos Indígenas. Declaración red de universidades indígenas interculturales de Abya Yala. Córdoba, Argentina, 2018.

\footnotetext{
5 En la Política de gestión intercultural de cooperación, solidaridad y complementariedad a nivel nacional e internacional de URACCAN, con perspectiva de género, se define ciudadanía intercultural como la capacidad de los pueblos indígenas, afro descendientes y mestizos autónomos de ejercer sus derechos individuales y colectivos, teniendo para ello acceso con equidad social a la educación, salud, bienestar material, trabajo y participación, en base a su propia manera de ver el mundo (Alta Hooker, Rectora URACCAN). La construcción de ciudadanía intercultural se ejerce promoviendo la participación real y efectiva de los hombres y mujeres de las comunidades y pueblos indígenas, afrodescendientes y mestizos costeños en la transformación de su entorno para acceder a un mejor nivel de vida. Para lograr esta participación efectiva es más que necesario identificar los factores estructurales que inciden en una desigual participación entre hombres y mujeres a lo interno de cada pueblo y entre los mismos, lo que permitirá la construcción de estrategias diferenciadas que hagan posible la verdadera consecución de ciudadanías interculturales de género (Política Intercultural de Género de URACCAN 2010).

La construcción de ciudadanía intercultural es un proceso de participación activa, autogestionaria y liberadora generando las oportunidades para que los pueblos y comunidades indígenas, afrodescendientes y mestizos costeños sean los actores claves de su propio desarrollo y del país. En el contexto específico de la Costa Caribe Nicaragüense, ciudadanía intercultural se equipara con el ejercicio pleno de los derechos autonómicos consignados en la Constitución de la República y en la ley 28.
} 
Hooker-Blandford, A. (2018). Universidades e instituciones de educación superior indígenas, interculturales, afrodescendientes y comunitarias en América Latina. En: Daniel Mato, coord., Educación Superior, Diversidad Cultural e Interculturalidad en América Latina. 299-322.

Mato, D. (2008). No hay saber "universal", la colaboración intercultural es imprescindible. Alteridades 18(35):101-116.

Mato, D. (2009). Contextos, conceptualizaciones y usos de la idea de interculturalidad. En: Miguel Ángel Aguilar y otros, (Edits.) Pensar lo contemporáneo: De la cultura situada a la convergencia tecnológica. Págs.: 28-50. Barcelona y México: Anthropos Editorial y Universidad Autónoma Metropolitana-Iztapalapa.

Mato, D. (2016). Educación Superior y Pueblos Indígenas en América Latina: Del “Diálogo de Saberes" a la construcción de modalidades sostenibles de "Colaboración Intercultural". Revista del CISEN Tramas/ Maepova 4(2): 71-94.

Mato, D. (2018). Educación Superior y Pueblos Indígenas y Afrodescendientes en América Latina. Aspectos sociales y normativos, experiencias, logros, aprendizajes y desafíos. En: Daniel Mato, coord. Educación Superior, Diversidad Cultural e Interculturalidad. Caracas: UNESCO-IESALC y Córdoba: Universidad Nacional de Córdoba.15-36.

Mato, D. (2018). Educación superior y pueblos indígenas: Experiencias, estudios y debates en América Latina y otras regiones del mundo. Revista del Cisen Tramas/Maepova, 6 (2), 41-65.

Ocoró-Loango, A., \& Silva, M. (2018). La Educación Superior y pueblos afrodescendientes en América Latina. En: Daniel Mato, coord., Educación Superior, Diversidad Cultural e Interculturalidad en América Latina. 323-350.

Sarango, L. (2009). Universidad Intercultural de las Nacionalidades y Pueblos Indígenas «Amawtay Wasi». Ecuador / Chinchaysuyu. En Daniel Mato, coord., Instituciones Interculturales de Educación Superior en América Latina. Procesos de construcción, logros, innovaciones y desafíos. Caracas: UNESCO- IESALC. 191-213.

Tercera Conferencia Regional de Educación Superior para América Latina y el Caribe. Declaración. Córdoba Argentina (2018).

URACCAN (2004). El Modelo Pedagógico de URACCAN. Managua: URACCAN.

URACCAN (2016). Proyecto Educativo de la Universidad de las Regiones Autónomas de la Costa Caribe Nicaragüense. Managua: URACCAN.

URACCAN (2018). Informe de Gestión Institucional DCE I Semestre. Managua: URACCAN.

URACCAN (2018). Universidad Comunitaria Intercultural. Perfil Institucional. Managua: URACCAN.

Zúñiga Muñoz, X. (2018). Persistencias coloniales, aperturas y desafíos para la educación superior en Centroamérica. En: Daniel Mato, coord. Educación Superior, Diversidad Cultural e Interculturalidad. 117-142. 